Mots. Les langages du politique

Discours de haine dans les réseaux socionumériques

Pierre Rosanvallon, Le siècle du populisme : histoire, théorie, critique

Paris, Seuil, 2020, 275 p.

Guillaume Grignard

\title{
OpenEdition
}

Journals

Édition électronique

URL : https://journals.openedition.org/mots/28193

DOI : $10.4000 /$ mots. 28193

ISSN : 1960-6001

Éditeur

ENS Éditions

Édition imprimée

Date de publication : 4 mars 2021

Pagination : 135-140

ISBN : 979-10-362-0306-0

ISSN : 0243-6450

Référence électronique

Guillaume Grignard, «Pierre Rosanvallon, Le siècle du populisme : histoire, théorie, critique », Mots. Les

langages du politique [En ligne], 125 | 2021, mis en ligne le 12 février 2021, consulté le 26 avril 2022.

URL : http://journals.openedition.org/mots/28193; DOI : https://doi.org/10.4000/mots.28193

Ce document a été généré automatiquement le 26 avril 2022

(c) ENS Éditions 


\section{Pierre Rosanvallon, Le siècle du populisme : histoire, théorie, critique}

Paris, Seuil, 2020, 275 p.

Guillaume Grignard

\section{RÉFÉRENCE}

Pierre Rosanvallon, Le siècle du populisme : histoire, théorie, critique, Paris, Seuil, 2020,

$275 \mathrm{p}$.

1 Dans le contexte où de nombreux pays européens sont soumis à une percée électorale de partis radicaux, appelés souvent rapidement "populistes », Pierre Rosanvallon propose un essai qui vise à conceptualiser le populisme en lui donnant des caractéristiques essentielles et généralisables à de nombreuses situations. Il faut saluer d'emblée l'ambition de l'auteur qui explique dès l'introduction les limites de la littérature actuelle, qui se focalise sur des contextes nationaux particuliers. Or, il y a «une réalité à théoriser» (p.11) et c'est l'ambition de l'auteur dans cet ouvrage : dégager, au-delà des particularismes, les caractéristiques essentielles du populisme.

2 L'auteur articule sa démonstration en trois temps : l'anatomie (partie I) relève certains concepts centraux des partis populistes. Le vocabulaire médical prend une grande force sémantique ici. P. Rosanvallon ausculte littéralement ces partis, en relevant les premières caractéristiques fondamentales. L'histoire (partie II) s'appuie sur l'étude de trois temporalités à la fin du $\mathrm{XIX}^{\mathrm{e}}$ et au début $\mathrm{du} \mathrm{Xx}^{\mathrm{e}}$ siècle, périodes dans lesquelles l'auteur dégage des moments populistes comparables à ceux de notre époque contemporaine. La critique (partie III) entame alors une discussion fondamentale sur les éléments marquants du populisme. L'ouvrage s'achève sur une conclusion qui propose une alternative pour réenchanter la démocratie.

D'emblée, le lecteur distingue aisément les motivations explicites et implicites de l'auteur. P. Rosanvallon apporte des éléments d'analyse du populisme en Europe et dans le monde. Mais, de manière plus latente, son livre parle surtout de démocratie, 
quitte à en faire un objectif d'écriture: démontrer les impasses des mouvements populistes et proposer une alternative pour renforcer la qualité de la démocratie. "L'esprit d'une alternative» (partie III, p. 243), qui fait office de conclusion, lève les derniers doutes du lecteur. L'ouvrage, dans lequel le sujet passe fréquemment du populisme à la crise de légitimité du système démocratique, offre ainsi une double lecture possible, s'inscrivant alors dans la continuité des travaux antérieurs de l'auteur (par exemple Rosanvallon, 2006 et 2015).

4 L'anatomie du populisme s'articule autour de six sections. «Le peuple-Un» (p. 27) discute d'un premier élément fondamental qui est celui de la reconstruction sociologique du peuple en une entité simplifiée, distinguant un «eux», les élites oligarchiques, contre un «nous », le peuple. L'auteur revisite la discussion sur la fin des classes sociales et argue que les mouvements populistes «entendent redonner une consistance sensible à l'invocation d'un peuple-Un devenu introuvable» (p. 34). En filigrane, ce thème rejoint celui de la séparation entre les $1 \%$ les plus riches et les $99 \%$ restants, qui sera centrale tout au long de l'ouvrage. La deuxième section discute des attaques populistes dirigées contre les démocraties "libérales-représentatives » (p.37). Pour les mouvements populistes, il s'agit à la fois de promouvoir des instruments de démocratie directe, comme l'usage du référendum, et de contester « le gouvernement des juges" (p.41), qui repose sur l'importance des cours constitutionnelles pour garantir les institutions démocratiques. Il s'agit là d'une discussion classique à propos de la démocratie libérale, que l'on retrouve dans certains ouvrages récents (par exemple Przeworski, 2019). La troisième section prolonge la réflexion sur le "peuple-Un » en discutant de l'appropriation du peuple par un leader. Se référant à des leaders historiques d'Amérique latine, comme le Colombien Jorge Eliécer Gaitán, P. Rosanvallon montre comment les leaders des mouvements populistes s'affirment comme représentant le peuple tout entier. La quatrième section aborde une thématique centrale qui est celle du protectionnisme économique. Si l'auteur ne l'aborde pas en profondeur dans la suite de l'ouvrage, d'après lui par défaut de compétence (p.170), il en propose ici une discussion historique remontant aux premiers travaux de Friedrich List. Cette section traite du déclassement et retrouve la thématique des écarts de richesses entre les $1 \%$ les plus riches et les $99 \%$ restants. La cinquième section traite d'un domaine en plein essor dans les sciences politiques: le rapport entre émotion et politique (par exemple Wahl-Jorgensen, 2018). L'auteur relie ce thème à la diffusion croissante des théories conspirationnistes et argue qu'il y aurait une " personnalité » populiste (p. 74) se caractérisant par une manière de recourir aux émotions populaires pour façonner une stratégie électorale. Enfin, la dernière section de cette première partie aborde la question des convergences des populistes de gauche et de droite. Alors que les deux courants partageraient une vision «dégagiste» des élites en place, ils différeraient, selon P. Rosanvallon, dans leur rapport à l'immigration et à la haine de l'autre, domaines qui seraient marqués par une franche différence perceptible dans l'histoire de ces mouvements. Cette anatomie propose donc six éléments de discussion qui se recoupent par moments et qui préparent une plus longue investigation dans la suite de l'ouvrage.

5 Dans la deuxième partie de l'ouvrage, plus historique, P. Rosanvallon étudie en profondeur trois moments populistes qui ont émaillé la vie politique internationale aux $\mathrm{XIX}^{\mathrm{e}}$ et $\mathrm{XX}^{\mathrm{e}}$ siècles. Usant de nombreuses références théoriques de l'époque, il montre dans la première section comment les écrits de Napoléon III peuvent prendre sens dans le débat sur le populisme contemporain. L'auteur revisite le thème du plébiscite et 
surtout celui du lien direct entre le peuple et «l'empereur»(p.100), lien qui s'accompagne d'une méfiance persistante à l'encontre des partis et des organisations. La presse fait également l'objet d'une vive méfiance pour cette même raison qu'il ne saurait y avoir aucun organe intermédiaire légitime entre le peuple et le souverain. Dans la section suivante, P. Rosanvallon traite avec intérêt de la question de la première mondialisation (1890-1914). Cela permet d'étudier le thème des inégalités et des dérives xénophobes envers les migrants, dans un contexte alors fort comparable à notre époque. L'auteur traite du mouvement boulangiste en France (p. 118), puis de la réaction américaine vis-à-vis de l'immigration chinoise (p.128), dans laquelle protectionnisme économique et racisme se confondaient. Ces analyses montrent la centralité de la question de l'ouverture des économies dans l'appréhension des mouvements populistes. Enfin, la dernière section historique, consacrée aux leaders d'Amérique latine $\mathrm{du} \mathrm{xx}^{\mathrm{e}}$ siècle, livre une analyse approfondie de la figure charismatique et émotionnelle du leader populiste. Tant Jorge Eliécer Gaitán que Juan Perón n'auront de cesse de s'affirmer comme étant au service du peuple. P. Rosanvallon fait suivre ces sections d'une transition vers la partie critique qui a pour but de conceptualiser les épisodes historiques en élargissant la vision du monde populiste. Si cette présentation historique n'a pas pour lui valeur d'explication, elle permet néanmoins de dégager certains éléments marquants du populisme, comme le rapport au peuple dans son ensemble ou la personnalité des dirigeants.

Dans la troisième partie, P. Rosanvallon se saisit des caractéristiques essentielles du populisme précédemment identifiées pour en apporter une critique. L'ouvrage laisse alors clairement entrevoir son projet implicite: fournir une réponse à la critique populiste de la démocratie contemporaine. Le référendum est d'abord abondamment discuté : d'après l'auteur, il diluerait la notion de responsabilité du corps politique, s'opposerait au principe de délibération et présenterait une tendance à conduire à une situation irréversible (l'auteur cite à de nombreuses reprises l'exemple du Brexit). La section suivante défend l'importance des cours d'arbitrage et critique la notion de "gouvernement des juges ». Elle explore aussi quelques thèmes susceptibles d'enrichir la démocratie actuelle, comme la question du tirage au sort, qui est également présentée dans sa dimension historique. La section suivante interroge la notion de peuple. L'auteur explore alors plus en profondeur la construction sociologique qui oppose les $1 \%$ les plus riches aux $99 \%$ restants. Enfin, la dernière section interroge le concept de "démocrature ", régime menant la démocratie vers une forme de pouvoir autoritaire. P. Rosanvallon illustre cette notion par l'irréversibilité qui voit de nombreux leaders populistes tenter d'augmenter le nombre de leurs mandats électifs. L'exemple d'Hugo Chavez est abondamment cité. «L'alternative » (partie III, p. 245) fait office de conclusion et développe ce que l'auteur nomme une «démocratie interactive ", qui inclut les thèmes d'une plus grande participation citoyenne au sein des instances politiques.

7 L'ouvrage de P. Rosanvallon est un projet ambitieux qui vise à saisir les caractéristiques générales et essentielles des phénomènes populistes. D'emblée, la double lecture qu'offre l'ouvrage stimule le lecteur autant qu'elle l'interroge. Si les débats sur la qualité de la démocratie sont aussi riches que dans les écrits précédents de l'auteur, on perd parfois le contact avec le populisme comme sujet premier. Le livre évoque en réalité davantage la question de la démocratie confrontée au populisme. C'est pourquoi 
il n'apporte peut-être pas d'éléments marquants pour appréhender le populisme au sein des démocraties contemporaines.

Si le lecteur reste ébloui par la passion de l'auteur pour son sujet et par son érudition historique, qui se manifeste avec éclat dans la deuxième partie de l'ouvrage, trois manquements méritent tout de même d'être soulignés. D'abord, P. Rosanvallon prend à notre avis trop de liberté avec un concept fondamental dans les sciences humaines, si bien théorisé par Max Weber, celui de la neutralité axiologique. L'auteur écrit en effet un livre contre le populisme et non sur le populisme. La nuance est centrale parce qu'elle explique les nombreux problèmes de rigueur scientifique qui entravent la démonstration de l'auteur. En effet, à de nombreuses reprises, P. Rosanvallon affirme des points en omettant d'argumenter son propos. Et cela dès le titre: peut-on raisonnablement, en 2020, parler du XxI ${ }^{e}$ siècle comme du « siècle du populisme » ? Dès sa première phrase ensuite : «le populisme révolutionne la politique du Xxi ${ }^{e}$ siècle ». Et enfin dans de nombreux passages du livre où l'auteur s'acharne tant sur le Brexit que sur la figure de Jean-Luc Mélenchon. Il y a dans ce livre un projet politique qui est louable dans le cadre d'un essai, mais problématique quand il est lu dans une perspective académique. Le propos, souvent excessif, ne s'inscrit pas toujours dans une logique de démonstration rigoureuse: sur quoi se fonde l'auteur, par exemple, pour évoquer le Brexit comme « une tragédie » (p. 171) ? Comment l'auteur justifie-t-il le fait que le populisme aurait réellement « révolutionné » la politique mondiale ? Si les gains électoraux de plusieurs partis radicaux interpellent, leur présence au pouvoir reste plutôt rare, car de nombreux pays sous gouvernement de coalition appliquent explicitement ou non une politique de cordon sanitaire (le cas de l'Allemagne ou de la Belgique, tant au niveau local que fédéral, est ici exemplatif). En outre, certaines victoires qualifiées de populistes par l'auteur, comme celle de Donald Trump aux ÉtatsUnis, s'expliquent par de nombreux facteurs bien éloignés du populisme. Par manque de rigueur, l'auteur appuie sa réflexion sur des postulats qui sont davantage des opinions personnelles que des vérités établies sur le plan scientifique.

9 La réflexion menée dans l'ouvrage manque par ailleurs d'un ancrage empirique solide. P. Rosanvallon explique avec conviction que tout phénomène populiste ne saurait être appréhendé qu'à la seule lecture de la réalité nationale dans laquelle il s'inscrit, ce qui rendrait toute tentative de généralisation impossible. Pourtant, le populisme tel que l'envisage l'auteur s'enracine bien quelque part. De manière implicite, l'auteur opère en effet une sélection parmi les exemples de populisme: le cas de la France est abondamment convoqué, ceux de l'Italie, des États-Unis ou du Royaume-Uni sont également cités. Mais rien n'est dit sur de nombreux cas tout aussi pertinents (les pays scandinaves, l'Allemagne, les Pays-Bas) qui proposent une version différente du problème sur le plan politique. L'auteur, en privilégiant l'exemple de certaines réalités nationales au détriment d'autres, sans apporter de justification, fait à nouveau preuve d'un manque de rigueur méthodologique. La Belgique représente par exemple un cas typique pour discuter de la fusion possible des populistes de droite et de gauche. En décembre 2019, le président du PTB-PVDA, parti de la gauche radicale, et celui du Vlaams Belang, parti d'extrême droite flamand, ont posé ensemble à la une du journal De Zondag (Le Soir, 2019), ce qui a déclenché un vif débat en Belgique.

10 Enfin, plusieurs des caractéristiques essentielles du populisme identifiées par l'auteur peuvent poser question, au sens où elles sont partagées par de nombreux partis politiques. La question du référendum, par exemple, est soutenue par un large spectre 
des forces politiques. De même que tous les partis jouent aujourd'hui sur le registre des émotions dans leur communication politique. Le discours hostile à la justice et aux médias est également répandu. Lors de la dernière campagne présidentielle en France, François Fillon, le candidat des Républicains, n'a eu de cesse de contester le gouvernement des juges et l'acharnement médiatique. En quoi ces éléments seraient-ils donc des caractéristiques spécifiques des partis populistes? Le projet économique protectionniste et souvent hostile aux institutions européennes apparaît plus propre à ces partis, il aurait sans doute mérité une plus vaste exploration.

11 Malgré ces réserves, l'ouvrage de P. Rosanvallon offre une perspective intéressante sur un sujet majeur des sciences politiques contemporaines. Ces critiques appellent à considérer son ouvrage comme un essai engagé pour la défense d'une certaine vision de la démocratie. Lorsqu'il est considéré dans cet esprit, l'ouvrage révèle tout son intérêt. La perspective historique offre au livre une réelle valeur bibliographique et tout chercheur qui s'interroge sur les partis populistes pourra trouver dans la pensée de l'auteur des éléments pour stimuler sa réflexion. Ces éléments permettent alors de dépasser les quelques lacunes méthodologiques de l'ouvrage pour s'approprier le sujet vers d'autres horizons.

\section{BIBLIOGRAPHIE}

PRZEWORSKI Adam, 2019, À quoi bon voter ?, S. Hirèche trad., Genève, Éditions Markus Haller.

Rédaction du journal Le Soir, 2019, «Peter Mertens (PTB) et Tom Van Grieken (Vlaams Belang) posent ensemble : une photo qui fait polémique », Le Soir, 22 décembre 2019.

ROSANVALLON Pierre, 2015, Le bon gouvernement, Paris, Seuil.

ROSANVALlon Pierre, 2006, La contre-démocratie : la politique à l'âge de la défiance, Paris, Seuil.

WAHL-JoRgEnSEn Karin, 2018, Emotions, Media and Politics, Cambridge, Medford, Polity Press.

\section{AUTEURS}

\section{GUILLAUME GRIGNARD}

Université libre de Bruxelles 